single use; and 4) using heat-based sterilization methods (eg, steam autoclave or dry-air oven) not liquid chemical germicides, to reprocess reusable needles and syringes.

FROM: Centers for Disease Control and Prevention. Improper infection control practices during employee vaccination programs-District of Columbia and Pennsylvania, 1993. M M WR 1993;42:969-971.

\section{First Reported Case of Patient-to- Patient Transmission of HIV in a Healthcare Setting}

In the December 18, 1993, issue of Lancet, Dr. Kerry Chant et al reported the results of an investigation in Australia that indicated apparent transmission of HIV from one patient to four other patients in a surgeon's office. Four female patients with risk factors for HIV underwent minor surgical procedures (eg, removal of skin lesions) on the same day in 1989. A male patient with known risk factors for HIV, who has died of AIDS since, also had surgery on the same day and is presumed to have been the source of infection to the other four patients. The surgeon, who has tested negative for HIV infection, performed these procedures without assistance.

Australian health officials believe that a breach in infection control precautions caused HIV to be transmitted from one patient to subsequent patients. Examples of such breaches in infection control include inadequate disinfection and sterilization of reusable instruments, reuse of needles or syringes designed for single use only, or improper technique for drawing blood and infection of medications. Although the precise event(s) that led to patient-to-patient transmission have not been identified to date, Australian health officials have said that an investigation has found that the doctor did not sterilize all his equipment. The investigation is continuing, and DNA viral sequencing studies are being performed.

\section{Surgeons Still Deficient in Hepatitis B Vaccination}

According to an abstract submitted to the First National Conference on Human Retroviruses and Related Infections, held December 12-16, 1993, in Washington, DC, the CDC conducted a seroprevalence survey of HIV HBV, and HCV infection among hospital-based surgeons in moderate to high HIV/AIDS incidence areas. The survey was voluntary and anonymous and included 770 (27\%) of 2,887 eligible surgeons. The participants reported practic- ing a mean of 7.8 years since 1978 and, in the past year, performing a mean of 174 operating room procedures and sustaining a mean of three percutaneous injuries. One $(0.14 \%)$ of 740 surgeons not reporting nonoccupational HIV risk factors was HIV seropositive. None of 20 surgeons reporting nonoccupational HIV risk factors were HIV positive. None of the participants not responding to the questions of nonoccupational risk factors was HIV positive.

One hundred twenty-nine surgeons had a pattern of HBV serologic markers indicating past HBV infection. Among participants, 418 (55\%) reported receiving $\geqslant 3$ doses of hepatitis B vaccine; of these, $88 \%$ had detectable levels of antiHBs. However, 199 surgeons (26\%) had not received hepatitis B vaccine, and of these, 105 (53\%) were susceptible to HBV infection. Seven surgeons were positive for anti-HCV

These results do not indicate a high rate of previously undetected HIV infection among surgeons who practiced in moderate to high HIV/AIDS incidence areas. In addition, a substantial percentage of surgeons are susceptible to HBV infection and need to be vaccinated.

FROM: Panlilio A, et al. Serosurvey of HIV, HBV, and HCV among hospital-based surgeons. Abstracts of the First National Conference on Human Retroviruses and Related Infections. December 12-16, 1993; Washington, DC. Abstract 536.

\section{Pediatric Emergency Departments -- Missed Opportunities for Measles Vaccination During Outbreaks}

The CDC's Division of Immunization reported the performance of two inner-city hospital pediatric emergency department (ED) immunization programs that were implemented during a measles outbreak. ${ }^{1}$ The two pediatric EDs were located in urban Chicago and served primarily an indigent minority population. As part of outbreak control, measles vaccine was provided free of charge to both hospital EDs by the local health department and specific procedures were developed for the vaccination programs, including a triage nurse to obtain a parental history of vaccination and a nurse specifically hired to administer vaccinations. The study, reported in a recent issue of the Journal of the American Medical Association, found that $59 \%$ of the vaccine-eligible patients seen in the EDs were not vaccinated.

Some of the factors that may have adversely affected the success of these vaccination programs included misperceptions by healthcare providers about valid contraindications to vaccination and less aggressive screening of older children for vaccination history 